\title{
Programa de Fortalecimiento PaRENTAL EN CONTEXTOS DE VULNERABILIDAD SOCIAL: UNA PROPUESTA DESDE EL ÁMBITO ESCOLAR
}

\section{Program OF PARENTAL STRENGTHENING IN CONTEXTS OF SOCIAL VULNERABILITY: A PROPOSAL FROM THE SCHOOL ENVIRONMENT}

\author{
Jael Vargas Rubilar*, Viviana Lemos ${ }^{* *}$ y María Cristina Richaud***
}

\author{
*Doctora en Psicología. Miembro de la Carrera del Investigador Científico del Consejo Nacional de Investigaciones \\ Científicas y Técnicas (CONICET) y Docente de la Universidad Adventista del Plata (UAP). \\ E-Mail: psicojael@gmail.com \\ ${ }^{* * *}$ Doctora en Psicología. Miembro de la Carrera del Investigador Científico del Consejo Nacional de Investigaciones \\ Científicas y Técnicas (CONICET) y Docente de la Universidad Adventista del Plata (UAP). \\ E-Mail: viviananoemilemos@gmail.com \\ ${ }^{* * *}$ Doctora en Psicología. Miembro de la Carrera del Investigador Científico del Consejo Nacional de Investigaciones \\ Científicas y Técnicas (CONICET) y Docente de la Universidad Adventista del Plata (UAP). \\ E-Mail: richaudmc@gmail.com \\ Grupo Vinculado al Centro Interdisciplinario de Investigaciones en Psicología Matemática y Experimental \\ Dr. Horacio J.A. Rimoldi (CIIPME). \\ Consejo Nacional de Investigaciones Científicas y Técnicas (CONICET). \\ Universidad Adventista del Plata (UAP). \\ Diamante, Provincia de Entre Ríos. República Argentina
}

\section{RESUMEN}

El trabajo que se informa destaca el rol relevante de la familia y del ejercicio adecuado de la parentalidad para el desarrollo humano. Dado que las competencias parentales estarían relacionadas con el contexto social, se describen las características de las familias en vulnerabilidad social, teniendo en cuenta los riesgos psicosociales a los que muchas veces están expuestas, las consideraciones culturales de lo que significa ser buenos padres y las propias experiencias de los progenitores o cuidadores en sus familias de origen. Se revisan diversas modalidades de programas de intervención familiar y parental, destacando la importancia del diseño, implementación y evaluación de estos programas en contextos de vulnerabilidad social. En este marco, y considerando que en Argentina existe un escaso desarrollo de programas de apoyo a las familias socialmente vulnerables, se presenta una propuesta de intervención aplicable en el ámbito escolar y dirigida a fortalecer las competencias parentales.

El programa propuesto, basado en el enfoque de la parentalidad positiva, integra elementos de los modelos de intervención educativo y comunitario, ya que se implementa en forma grupal, mediante encuentros expositivo-participativos y en coordinación con la escuela a la que asisten los hijos de los beneficiarios. Como conclusión y en función de la experiencia obtenida por las interventoras y la observada en las madres participantes, se presenta una reflexión sobre las contribuciones de la intervención propuesta y los desafíos pendientes para futuras aplicaciones del programa.

Palabras clave: Parentalidad; Educación parental; Intervención; Relación familia - escuela; Vulnerabilidad social. 


\section{ABSTRACT}

This study stresses the significant role of the family and a proper parenting performance for human development. Parenting in itself is very complex, since it demands a balanced combination of several essential factors such as affection, communication, discipline, and the autonomy granted to children. Given the fact that parental skills would be related to a specific social context, a description of the characteristics of families in situations of social vulnerability is provided. Likewise, it is noteworthy that parenting development would depend on those cultural considerations implicated in the concept of being good parents, and the parents or caregivers' own experiences of upbringing, temperament and resilience. Subsequently, the main modalities of family intervention programs are reviewed, highlighting the importance of design, implementation and evaluation of the former in contexts of social vulnerability. Within this framework, and considering that in Argentine there are very few programs supporting families in social vulnerability, the main aim of this research is to present an intervention proposal to strengthen parenting skills applicable in the school setting. The proposed program, which is based in the approach of positive parenting, integrates elements from educational and community intervention models, since it is performed in groups, by means of participative and expositive meetings and in coordination with the school attended by the children of the project participants. The proposed intervention was based on two basic areas: (a) direct intervention for parents: the promotion of parenting skills (i.e., attachment, parental empathy, parenting style, positive communication and support networks) and (b) indirect intervention for children: training parents in strategies to promote children's development of socio-emotional resources (e.g., positive emotions, prosocial behavior, etc.). The intervention was carried out in a school within a context of social vulnerability in the province of Entre Ríos (Argentina). The organization of the meetings was conducted by an interdisciplinary team (i.e., psychologists and educational psychologists).

The topics addressed were agreed with the participants based on their interests and needs; a total amount of 30 meetings of 60 to 90 minutes each were carried out. The themes were as follows: pa- rental and child self-esteem, appropriate expression of affection, positive communication and conflict resolution, positive emotions, the strengthening of cognitive resources, prosocial behavior, social skills, family, parental and child resilience, social support networks to families and family-school relationships. The main approach tech-niques used were: (a) information exposure, (b) peers' feedback and co-reflection, (c) modeling of attitudes and alternative behaviors, (d) task assignments and proposals for action, and (e) interviews after workshops. The research findings are related to those found by other intervention programs made with parents and caregivers in other countries. These preliminary results show that parenting skills, the type of relationship and even the attachment with the children could be modified through the implementation of programs of parental strengthening. Furthermore, the results showed that the program could also help to improve family-school relationships. In some cases, the intervention implemented aroused the interest of the participants in the schooling situation of their children, and at the same time it improved the communication among parents, teachers, and the people in charge of the school. The exploratory results of this proposal of intervention clearly show the need to support and implement these specific kinds of programs, which aim to strengthen the resources of parenting skills and encourage a healthy development of children. Based on the experience got from interventionists and participating mothers, a final consideration about the contributions of the proposed intervention and the possible future challenges observed after the implementation of the program is presented.

Key words: Parenting; Parental education; Intervention; Family-school relationship; Social vulnerability.

\section{INTRODUCCIÓN}

El ámbito familiar ha sido ampliamente reconocido como uno de los factores más relevantes para el desarrollo humano en todas sus etapas (Bronfenbrenner \& Evans, 2000; 
Muñoz Silva, 2005; Sanders \& Morawska, 2010; Vargas Rubilar, 2009). Aunque los niños reciben influencias desde diversos ámbitos (i.e., escuela, grupo de pares, etc.), el entorno familiar es fundamental para el crecimiento sano debido a que es el primero, el más persistente e intenso a nivel afectivo y en el cual se desarrollan los vínculos iniciales y necesarios para la supervivencia (Grusec, 2002; Palacios, 1999). Si bien las funciones familiares pueden cambiar a lo largo del ciclo vital de una persona, la familia de origen sigue repercutiendo en la vida de sus miembros (Muñoz Silva, 2005). En efecto, diversos especialistas precursores en el tema (Baumrind, 1971, 1978; Grusec, 2002; Maccoby, 1992) han señalado que la familia es el entorno social por excelencia en el que los niños adquieren las primeras habilidades y hábitos, fortaleciendo su autonomía y otras conductas cruciales para la vida.

Existe amplia evidencia sobre los efectos que tiene el contexto familiar en el desarrollo afectivo, social y cognitivo de los hijos, en especial en las primeras etapas del desarrollo humano (infancia y niñez) y su repercusión en fases posteriores (e.g., Barudy \& Dantagnan, 2005, 2010; Baumrind, 1971, 1978; Bowlby, 1958; Bronfenbrenner \& Evans, 2000; Gracia \& Musitu, 2000; Grusec \& Goodnow, 1994; Izzedin Bouquet \& Pachajoa Londoño, 2009; Krumm, Vargas Rubilar \& Gullón, 2013; Maccoby, 1992; Muñoz Silva, 2005; Richaud de Minzi, 2007; Richaud, Lemos \& Vargas Rubilar, 2014; Vargas Rubilar, 2009; Winnicott, 2006, entre otros). La mayoría de los estudios concuerda en que una de las funciones más importantes de la familia es la adecuada satisfacción de las necesidades socioafectivas y cognitivas de los hijos (Barudy \& Dantagnan, 2010; Gracia \& Musitu, 2000; Muñoz Silva, 2005; Palacios \& Rodrigo, 1998; Richaud de Minzi, 2007; Vargas Rubilar, 2009, 2014). Sin embargo, en la cotidianeidad pocos padres pueden acceder a dicha información, comprenderla y utilizarla de forma apropiada para guiar y / o modificar sus actitudes, creencias y prácticas de crianza en beneficio del desarrollo de sus hijos e hijas (Vargas Rubilar \& Oros, 2011).
Si bien los agentes socializadores y los ámbitos de socialización pueden ser diversos (e.g., escuela, barrio, etc.), el énfasis de la bibliografía ha sido puesto en el ambiente familiar, y especialmente en el rol de los padres. En este sentido, la función de crianza o la parentalidad social ha sido definida como las competencias, los conocimientos, las creencias, las pautas y las actitudes que los padres y madres asumen en relación a la socialización de sus hijos (Barudy \& Dantagnan, 2010; Eraso, Bravo \& Delgado, 2006). El ejercicio de la parentalidad implica en sí mismo una gran complejidad, ya que demanda la conjunción equilibrada de diversos factores indispensables: la sensibilidad y respuesta oportuna ante las necesidades de los hijos, la aceptación incondicional de la individualidad y la expresión apropiada del afecto, a la vez que el control o supervisión adecuados en relación a cada etapa evolutiva del niño (Barudy \& Dantagnan, 2010; Cirillo, 2012).

La función de crianza se lleva a cabo desde diferentes escenarios socioculturales que influyen en su desarrollo. La parentalidad es asimilada no como un aprendizaje formal, sino a través de la inmersión de relaciones interpersonales dentro de un determinado grupo social y cultural (Martín Quintana et al., 2009).

\section{FAMILIAS EN CONTEXTOS DE VULNERABILIDAD SOCIAL}

A lo ya señalado, debe agregarse la consideración de que el potencial del desarrollo psicosocial humano y familiar se encuentra estructuralmente limitado por las características socioambientales y económicas del entorno de vida cotidiano (Barudy \& Dantagnan, 2005; Bronfenbrenner \& Evans, 2000). En este sentido, Barudy y Dantagnan (2010) señalan que existen

"investigaciones que muestran el papel crítico de un ambiente social adverso para la salud y el desarrollo humano en general, y el de la parentalidad, en particular" (p. 378).

Dichos autores reconocen como eje principal factores intrapersonales (competencias pa- 
rentales), pero enfatizan el papel nocivo que pueden tener algunos entornos sociales. Muchas familias deben enfrentarse a diario a contextos desfavorables, con múltiples factores de estrés, consecuencias de la pobreza y de la exclusión social (Barudy \& Dantagnan, 2010; Gómez, Muñoz \& Haz, 2007).

En esta dirección, algunos organismos internacionales (CEPAL / CELADE, 2002) han definido a la vulnerabilidad social como una combinación de eventos, procesos o rasgos que constituyen adversidades potenciales para el ejercicio de los distintos tipos de derechos ciudadanos o el logro de los proyectos de las comunidades, los hogares y las personas. La vulnerabilidad social sería entonces la incapacidad de respuesta frente a la materialización de dichos riesgos y la inhabilidad para adaptarse a las consecuencias de dicha materialización.

Rodrigo, Máiquez, Martín y Byrne (2008, p. 42) han definido a las familias en vulnerabilidad psicosocial como

"aquellas en las que los responsables del cuidado, atención y educación del menor, por circunstancias personales y relacionales, así como influencias adversas de su entorno, hacen abandono de sus funciones parentales o realizan un uso inadecuado de las mismas, comprometiendo el desarrollo del niño, pero sin alcanzar la gravedad que justifique una medida de amparo, en cuyo caso se considera la separación del menor de su familia."

Por otra parte, un estudio reciente (Lecannelier, Flores, Hoffmann \& Vega, 2010) postula que el despliegue de competencias parentales estaría íntimamente ligado al medio social donde los procesos parentales se desarrollan, siendo sensibles al riesgo psicosocial (e. g., extrema pobreza, consumo excesivo de alcohol y drogas, ambientes delictuales, dinámicas de violencia intrafamiliar y maltrato), a las consideraciones culturales de lo que significa ser 'buenos padres' y a las propias experiencias de apego, temperamento y factores resilientes de los progenitores o cuidadores. De manera afín, Bornstein, L. y Bornstein, M.H. (2010) mencionan que en los grupos familia- res económicamente menos favorecidos y de alto riesgo, los factores ambientales (e.g., inseguridad, problemas económicos, bajo acceso a servicios, etc.) podrían tener un gran peso en el desarrollo del estilo de crianza de los padres.

Particularmente en la crianza de los hijos, Barudy y Dantagnan (2010) estiman que las funciones parentales más importantes (i.e., afectiva, socializadora y educativa) se encuentran debilitadas o disminuidas en la mayoría de los padres o cuidadores que viven en contextos de riesgo psicosocial. Las investigaciones desarrolladas en este tópico (Gómez et al., 2007; Pérez Padilla, Menéndez AlvarezDardet \& Hidalgo, 2014) indican que los padres de contextos socialmente vulnerables tienden a experimentar altos niveles de estrés con respecto a su rol de crianza, siendo muchas veces incapaces de responder satisfactoriamente las necesidades de sus hijos. Consecuentemente, los padres requieren muchas veces apoyo social externo y la colaboración de los diversos servicios sociales, aunque no siempre soliciten ayuda oportunamente (Ciri1lo, 2012; Pérez Padilla et al., 2014).

Aunque la intervención con estos grupos sociales no es reciente, sólo a partir de la década de los años 80 se empieza a comprender que las familias en vulnerabilidad social necesitan modelos de intervención centrados en el fortalecimiento de sus recursos y en la preservación familiar, desde una perspectiva más preventiva y positiva (Jiménez, 2009; Walsh, 2004). En este sentido, es destacable el rol que adquirió el enfoque del apoyo social en la intervención con familias y padres de contextos socialmente vulnerables, especialmente en tópicos como las redes formales de apoyo social o los sistemas de apoyo comunitario (Gracia, 1997; Gracia \& Herrero, 2006). Estos programas de fortalecimiento parental han sido considerados fuentes de apoyo social externo y al mismo tiempo, fortalecedores de redes informales y recursos parentales e intrafamiliares. Paralelamente, otras investigaciones en el área (Rodrigo \& Byrne, 2011) han mostrado que este apoyo percibido podría reducir el estrés parental generado por las condiciones adversas de vida 
y fomentar estrategias de afrontamiento más adecuadas en contextos socialmente vulnerables.

\section{MODELOS DE INTERVENCIÓN FAMILIAR}

Los programas de apoyo a grupos familiares proporcionan el soporte que fortalece los recursos psicosociales de los adultos en su rol de padres o cuidadores (Weissbourd \& Kagan, 1989). La mayoría de estos programas comparten como objetivo principal la prevención y el abordaje de una diversidad de problemas socio-familiares, que se encuentran asociados a la falta de apoyo social y educación de los padres como principales responsables de las familias (Gracia, 1997; Vargas Rubilar \& Oros, 2011).

En lo que se refiere a la intervención psicológica, existen diversos modelos y enfoques de abordaje familiar. En este sentido, algunos autores (Máiquez Chaves \& Capote Cabrera, 2001) han utilizado el término modelo para referirse a la

“descripción de diseños, estructuras o componentes de un proceso de intervención, es decir, plan guía de actuación” (p.189).

El término enfoque, en cambio, ha sido utilizado para describir la orientación teórica que subyace a determinado modelo.

Para Máiquez Chaves y Capote Cabrera (2001) los principales modelos en intervención familiar son: (a) el modelo clínico, (b) el educativo y (c) el comunitario. En el modelo clínico las intervenciones terapéuticas y / o educativas pueden ser individuales o con el grupo familiar, dependiendo del enfoque del terapeuta. El modelo educativo se focaliza en la enseñanza de comportamientos y habilidades parentales. Algunas de estas intervenciones pueden tener un propósito asistencial y / o preventivo, en tanto que procuran satisfacer necesidades actuales de la familia y advertir problemas futuros que puedan presentarse. Esta modalidad de intervención generalmente se dirige a grupos más que a individuos en particular.
El modelo social comunitario, en cambio, tiene como objetivo principal

"promover el cambio hacia un mayor bienestar psicológico y social" (Máiquez Chaves \& Capote Cabrera, 2001, p. 194).

Este tipo de abordaje puede contribuir a la producción de cambios en las actitudes y comportamientos individuales o en la calidad de las relaciones interpersonales, así como en el entorno social comunitario de los beneficiarios. Se consideran las potencialidades y capacidades de los grupos familiares y su entorno social como los principales recursos a trabajar.

Muchas de las intervenciones familiares comunitarias se basan en el enfoque teórico ecológico de Bronfenbrenner (1979, 1989). Desde la perspectiva ecológica, la relación del sistema familiar y su entorno es mutua y permanente. De esta manera, la familia y los sistemas extrafamiliares (i.e., la escuela, el trabajo, el barrio, etc.) establecen un proceso continuo de adaptación mutua (Bronfenbrenner, 1989).

En España y en algunos países latinoamericanos se realizaron revisiones teóricas y evaluaciones (en menor grado) sobre los efectos de los programas de intervención familiar y parental en sus diversas modalidades con buenos resultados (ver Cuadro 1). Un buen número de dichos estudios teóricos y empíricos sobre el abordaje con familias en contextos de vulnerabilidad social (Gómez, Cifuentes \& Ortún, 2012; Gómez \& Haz, 2008; Gó- mez, Muñoz \& Santelices, 2008; Trivette \& Dunst, 2010) ha sugerido como más efectivo el modelo social comunitario desde el enfoque teórico ecológico (Bronfenbrenner, 1979, 1989). Asimismo, el modelo educativo ha sido muy aplicado en programas de entrenamiento para padres o escuela para padres (Bartau Rojas \& de la Caba Collado, 2009; Pérez Campos, 2005; Rey, 2006). Por otra parte, algunos programas han integrado ambos modelos (Hidalgo García et al., 2009; Kotliarenco, Muñoz, Gómez \& Armijo, 2009).

En el contexto expuesto, y teniendo en cuenta la escasez de desarrollo, evaluación y 
difusión sistemática de programas de apoyo a la familia y / o fortalecimiento parental en Argentina, el principal objetivo del trabajo que se informa es presentar una propuesta de intervención en contextos de vulnerabilidad social aplicable al ámbito escolar.

\section{UN PUENTE ENTRE FAMILIA Y ESCUELA: UN PRO- GRAMA PARA PADRES EN EL ÁMBITO ESCOLAR}

El programa de fortalecimiento parental propuesto ha sido denominado Un puente entre familia y escuela, debido a que se aplica en el ámbito escolar y es parte de un programa mayor, uno de cuyos objetivos es fortalecer la vinculación familia - escuela.

Dicha intervención se diseñó e implementó en el marco del proyecto de evaluación y fortalecimiento de recursos psicológicos en niños Sin afecto no se aprende ni se crece (Richaud, 2013) teniendo como principal objetivo la promoción y el fortalecemiento de recursos lingüísticos, socioafectivos y cognitivos de niños de contextos socialmente vulnerables en el ambiente escolar.

La intervención propuesta está centrada en dos ejes básicos: (a) la intervención directa para los padres: la promoción de las competencias parentales (i.e., apego, empatía parental, estilo de crianza, comunicación positiva y redes de apoyo) (Barudy \& Dantagnan, 2010) y (b) la intervención indirecta para los hijos: la formación de los padres en estrategias para la promoción del desarrollo de recursos socioemocionales en los hijos (e.g., autoestima, emociones positivas, conducta prosocial, etc.). Si bien se recomienda comenzar con la intervención en las primeras etapas del desarrollo (i.e., primera infancia), realizar el abordaje al interior de los hogares podría resultar invasivo y contraproducente. Por lo tanto, se consideró más natural y apropiado el ámbito escolar. La intención de integrar la intervención con los niños al curriculum y a la vida escolar en general, era que el fortalecimiento de recursos psicológicos no se interrumpiera cuando los niños regresaban a sus casas, sino que por el contrario continuara a través del fortalecimiento cotidiano por parte de sus padres. Para ello, era necesario comenzar el trabajo con las historias de crianza, las creencias y los estilos educativos de los propios padres, para luego enseñarles cómo continuar la intervención a realizar con los niños, en la casa.

El diseño se basó en una extensa revisión de enfoques y modelos de intervención psicosocial, privilegiando el estudio de los de intervención con familias en vulnerabilidad y / o riesgo social y fueron los siguientes: los principios de la parentalidad positiva (Rodrigo López et al., 2009), el fortalecimiento de las competencias parentales (Barudy \& Dantagnan, 2005, 2010), el apoyo social en la intervención comunitaria (Gracia, 1997; Gracia \& Herrero, 2006), los principios de la resiliencia familiar y parental (Barudy \& Marquebreucq, 2005; Gómez \& Kotliarenco, 2010; Walsh, 2004), la vinculación familia y escuela (Bolivar, 2006; García-Bacete, 2003) y la coordinación con las redes sociales comunitarias (Barudy \& Dantagnan, 2010).

El programa integra elementos de los modelos educativo y comunitario, ya que se implementa en forma grupal mediante encuentros expositivo-participativos, en coordinación con la escuela a la que asisten los hijos de los padres participantes. Estos encuentros de fortalecimiento parental se plantearon como espacios de intercambio de experiencias, vivencias y conocimientos entre los profesionales y los participantes. Desde esta perspectiva, esta modalidad de abordaje también es experiencial ya que favorece la co-construcción de conocimientos episódicos y cotidianos en los beneficiarios (Martín Quintana et al., 2009). La intervención grupal también promueve el apoyo social informal de otros padres que viven en el mismo contexto social y por lo tanto están expuestos a situaciones similares.

Los temas de los encuentros fueron construidos con los participantes, es decir, se les dio protagonismo principal a los padres, ya que no se impuso una agenda temática, se propuso que ellos la armaran. Así se comenzó realizando una planificación anual en función de la necesidad manifestada por los participantes en las primeras sesiones. A los temas sugeridos por los padres, se fueron agregando 
otros basados en experiencias previas de estudios específicos de intervención con padres. Así, se abordaron las siguientes temáticas: (1) autoestima parental e infantil, (2) expresión adecuada del afecto, (3) comunicación positiva y resolución de conflictos, (4) emociones positivas, (5) fortalecimiento de recursos cognitivos, (6) conducta prosocial, (7) habilidades sociales, (8) resiliencia familiar, parental e infantil, (9) redes de apoyo social a familias y (10) vinculación familia-escuela.

La intervención se realizó en una escuela de contexto socialmente vulnerable de la provincia de Entre Ríos (Argentina). Inicialmente, se analizó el estrato social de las familias participantes, las cuales se situaron en estratos de nivel 5 (pobreza extrema) y nivel 4 (pobreza relativa) según la clasificación de la Escala Graffar (Méndez Castellano \& Méndez, 1994). En lo que respecta a la escolaridad de los padres, la mayoría contaba con instrucción básica y un bajo número de ellos tenía estudios secundarios completos. Si bien participó un número importante de padres de manera irregular, sistemáticamente asistieron entre 10 y 15 padres (madres en su mayoría), lo cual es adecuado para este tipo de intervenciones, ya que permite la interacción entre los participantes, propia de los talleres experienciales.

Un equipo interdisciplinario (i.e., psicólogos y psicopedagogos) tuvo a su cargo la coordinación de los encuentros. En el marco del programa mencionado se realizaron en total 30 encuentros de 60 a 90 minutos cada uno, asociados a la concurrencia de los padres al retirar los boletines de calificaciones y otras actividades escolares de sus hijos. Los encuentros se realizaron con una frecuencia mensual en el primer año y quincenal en el segundo, a fin de que en las semanas posteriores al taller, los padres pudieran entrevistarse con el equipo (ver Cuadro 2).

Las principales técnicas de abordaje utilizadas fueron las siguientes:

1.- La exposición de información consistió en brindar información relevante a los padres sobre crianza y desarrollo infantil. Por ejemplo, en el encuentro sobre disciplina positiva se presentó la importancia de conside- rar formas de control y monitoreo parental alternativas al maltrato, adecuadas a la etapa evolutiva de los hijos.

2.- La retroalimentación con pares y co-reflexión se refiere a una instancia de reflexión compartida, destinada a repensar conjuntamente el modo de llevar a cabo la función de crianza. Durante esta instancia, los padres e interventores intercambian y analizan sus conocimientos, creencias, actitudes y prácticas frente a situaciones cotidianas de la relación con sus hijos.

3.- El modelado de actitudes y comportamientos alternativos consiste en la presentación de conductas alternativas a las consideradas inadecuadas en la relación paterno-filial cotidiana. Se abordaron principalmente actitudes y conductas referidas a la expresión del afecto, la autonomía otorgada y la disciplina aplicada a los hijos.

4.- La asignación de tareas y propuestas de acción se refiere a actividades prácticas realizadas y supervisadas en las sesiones para consolidar lo trabajado en los talleres y promover su aplicación en el ámbito familiar. Por ejemplo, en el taller de fortalecimiento de la autoestima se trabajó por escrito la identificación de los aspectos positivos de los padres sobre su rol parental. Dicha actividad resultó beneficiosa para afianzar la agencia parental (ver Vargas Rubilar \& Oros, 2011).

5.- Entrevistas individuales con los psicólogos del equipo y posteriores a los encuentros solicitadas por los miembros participantes para responder consultas personales difíciles de atender en las sesiones grupales, especialmente por motivos éticos y de tiempo.

CONTRIBUCIONES Y DESAFÍOS DEL PROGRAMA DE INTERVENCIÓN PROPUESTO

Luego de la intervención se analizó la información obtenida desde la perspectiva de las madres participantes del programa y de sus respectivos hijos lo que evidenció resultados alentadores (Vargas Rubilar, Oros \& Richaud, inédito). El impacto positivo en las familias como contribución de este tipo de programas 
de fortalecimiento para padres también ha sido documentado en estudios de Chile (Gómez et al., 2012; Gómez \& Muñoz, 2012; Gómez et al., 2008), Estados Unidos (Sanders \& Ralph, 2004) y España (Bartau Rojas \& de la Caba Collado, 2009; Hidalgo et al., 2009; Peña et al., 2014; Rodrigo \& Byrne, 2011).

Con respecto al contexto natural de realización del programa (i.e., la escuela), se observó que éste también podría colaborar con una mejor interacción familia-escuela. En este sentido, la intervención realizada activó el interés de los participantes en la situación escolar de sus hijos y al mismo tiempo mejoró la comunicación entre los padres, docentes y directivos de la escuela, tal como quedó manifestado con la cantidad de comentarios positivos expresados por las madres participantes, que antes de la intervención eran inexistentes (Vargas Rubilar, Oros \& Richaud, inédito). Dicho cambio es sumamente relevante ya que la implicación de los padres puede favorecer la efectividad de la educación escolar (Rivas Borrel \& Ugarte Artal, 2014). En efecto, en la realidad cotidiana antes de la intervención se observaba un fuerte quiebre entre las instituciones socializadoras básicas: familia y escuela, de acuerdo con lo comentado por otros autores (Bolivar, 2006; García-Bacete, 2003; Rivas Borrel \& Ugarte Artal, 2014). En este sentido se encontró que la participación conjunta de las familias y de la comunidad educativa puede favorecer la formación positiva de los alumnos (Rivas Borrel \& Ugarte Artal, 2014).

A partir de lo observado durante la aplicación del programa puede señalarse que el proceso de convocatoria y asistencia a este tipo de intervención es y será uno de los principales desafíos observados. Muchos padres y madres no solicitan colaboración en la crianza de sus hijos, ni para mejorar su situación actual. Es posible que algunos padres se encuentren en tal estado de indefensión (depresión, alcohol, drogas, privación de la libertad, etc.) que no tengan la voluntad o la posibilidad de pedir ayuda. Estos problemas ya han sido señalados por especialistas en intervención parental cuando destacan la dificultad que tienen los padres para acercarse a estas instancias de participación para mejorar su relación con los hijos. Esto puede deberse a sentimientos de vergüenza, miedo a la crítica y a la sanción social, y principalmente a la incapacidad cultural para creer en el apoyo social, más allá de la ayuda asistencial a través de subsidios y elementos materiales que aportan los gobiernos (Cirillo, 2012; Cirillo \& Cipolloni, 1994). En este sentido, la convocatoria no implicó la necesidad de los padres de pedir ayuda sino que se trató de hacerlo con naturalidad, incluyéndola en las actividades escolares de sus hijos. Si bien esta modalidad fue exitosa para el acercamiento de los padres al programa, no lo fue tanto para retenerlos durante todos los encuentros.

A pesar de las dificultades que conlleva el diseño, la implementación y evaluación de programas de apoyo psicosocial a familias $\mathrm{y} / \mathrm{o}$ entrenamiento parental en contextos de riesgo psicosocial, éstos han mostrado resultados alentadores (Gómez et al., 2012; Peña, Máiquez \& Rodrigo, 2014). Diversos estudios analizados sobre intervenciones destinadas a mejorar la parentalidad, así como la propuesta de este trabajo (i.e., apego, competencias, estilos, creencias y prácticas parentales) aseguran que los basados en enfoques como el aprendizaje social y en principios cognitivos conductuales han demostrado ser efectivos (Sanders \& Morawska, 2010).

A partir de la experiencia realizada como interventoras e investigadoras se puede afirmar que los abordajes que tienen como propósito incrementar la sensibilidad parental, estilos y prácticas positivas y brindar información relevante con respecto a la crianza infantil (psicoeducación), promueven cambios positivos en las familias de contextos con vulnerabilidad social. Estos abordajes permitirían a los padres percibir a sus hijos de una manera menos distorsionada por las propias creencias e historia en su familia de origen, facilitando la resiliencia infantil, parental y familiar (Vargas Rubilar, 2014; Walsh, 2004).

En Argentina, como en muchos países de Latinoamérica, aún no se cuenta con el apoyo suficiente de políticas sociales, ni de programas de intervención destinados a promover la parentalidad positiva en contextos social- 
mente adversos. Los resultados exploratorios de la presente propuesta de intervención y las evaluaciones alentadoras de otros estudios ya referidos, muestran claramente la necesidad de apoyar e implementar este tipo de programas específicos, dirigidos a fortalecer los recursos parentales y repercutir en el desarrollo saludable de los hijos.

Otra tarea pendiente es la inclusión de estrategias para promover el fortalecimiento de recursos cognitivos, tanto en los padres como en los hijos (Vargas Rubilar \& Arán Filippetti, 2014). En Argentina se implementó una intervención para fortalecer recursos cognitivos de niños escolarizados en el marco del Programa Sin afecto no se aprende ni se crece (Richaud, 2013; Richaud \& Arán Filippetti, en prensa) con buenos resultados en los niños. Se estima que los resultados positivos aumentarían considerablemente si estos recursos se fortalecieran sistemáticamente desde el contexto familiar.
Si bien se enfatizó la necesidad de abordajes en contextos socialmente vulnerables, es importante destacar que otros estratos sociales (i.e., medios y altos) no están exentos de presentar factores que constituyan riesgos en el proceso de crianza de los hijos. En este sentido, Barudy y Dantagnan (2010) señalan que

"en los entornos caracterizados por la acumulación de las riquezas materiales se presenta el riesgo de transformar las relaciones en formalismos, que privan a los hijos de la afectividad, la educación y el apoyo social que necesitan" (p. 378).

Desde esta perspectiva, el programa propuesto debería adaptarse a las necesidades de cada grupo social, privilegiando la promoción de una parentalidad más positiva en beneficio de padres e hijos. 
CUADRO 1

PROGRAMAS PARA PADRES DESARROLLADOS EN ESPAÑA Y LATINOAMÉRICA

\begin{tabular}{|c|c|c|}
\hline País & Modelo de intervención & Estudio \\
\hline España & $\begin{array}{l}\text { Educativo } \\
\text { Comunitario }\end{array}$ & $\begin{array}{l}\text { Bartau Rojas \& Caba Collado (2009) } \\
\text { Torío López et al. (2010) } \\
\text { Martín Quintana et al. (2009) } \\
\text { Hidalgo García et al. (2009) } \\
\text { Arruabarrena \& De Paul (2002) } \\
\text { Rodrígo \& Byrne (2011) }\end{array}$ \\
\hline Chile & Comunitario y Educativo & $\begin{array}{l}\text { Gómez \& Haz (2008) } \\
\text { Gómez, Muñoz \& Santelices (2008) } \\
\text { Gómez, Cifuentes \& Ortún (2012) }\end{array}$ \\
\hline Brasil & Comunitario & $\begin{array}{l}\text { Sousa, Ribeiro \& Rodrigues (2006) } \\
\text { Pereira, D`Affonseca \& Williams (2012) }\end{array}$ \\
\hline Colombia & Educativo & Rey (2006) \\
\hline México & Educativo & $\begin{array}{l}\text { Nuño-Gutiérrez et al. (2006) } \\
\text { Pérez Cárdenas \& Fernández Benítez (2005) }\end{array}$ \\
\hline
\end{tabular}




\section{CUADRO 2}

Descripción del Programa Un Puente entre Familia y Escuela (Adaptado de Vargas Rubilar \& OROS, 2011)

\begin{tabular}{|c|c|}
\hline Objetivo general & $\begin{array}{l}\text { Fortalecer las competencias, estilos y prácticas parentales positivas en contextos de } \\
\text { vulnerabilidad social. }\end{array}$ \\
\hline Objetivos específicos & $\begin{array}{l}\text { - Estimular las prácticas de aceptación y apoyo incondicional hacia los hijos. } \\
\text { - Ayudar a los padres y cuidadores a identificar y satisfacer las necesidades de sus } \\
\text { hijos. } \\
\text { - Promover la expresión adecuada de sentimientos y emociones. } \\
\text { - Dar a conocer diferentes modos de comunicación familiar, resaltando la importan- } \\
\text { cia de un diálogo abierto y emocionalmente positivo. } \\
\text { - Instruir en modos de control asertivo, previniendo las diversas formas de maltrato } \\
\text { infantil y negligencia parental. } \\
\text { - Fomentar el respeto por la autonomía del niño. } \\
\text { - Potenciar una mejor vinculación familia-escuela. }\end{array}$ \\
\hline Enfoque & Ecológico o ecosistémico \\
\hline Modelo de Intervención & $\begin{array}{l}\text { Educativo } \\
\text { Comunitario }\end{array}$ \\
\hline Modalidad & Intervención grupal experiencial-participativa \\
\hline Técnicas & $\begin{array}{l}\text { - Exposición de información } \\
\text { - Retroalimentación con pares y co-reflexión } \\
\text { - Modelado y propuestas de acción } \\
\text { - Asignación de tareas } \\
\text { - Entrevistas posteriores al taller }\end{array}$ \\
\hline Secuencia metodológica & $\begin{array}{l}\text { - Evaluación pre-intervención } \\
\text { - Aplicación del programa } \\
\text { - Evaluación post-intervención }\end{array}$ \\
\hline Beneficiarios & $\begin{array}{l}\text { - Padres, madres y cuidadores } \\
\text { - Niños de edad escolar }\end{array}$ \\
\hline Contexto de aplicación & Ámbito escolar \\
\hline
\end{tabular}




\section{REFERENCIAS BIBLIOGRÁFICAS}

Arruabarrena I. \& De Paul, J. (2002). Evaluación de un programa de tratamiento para familias maltratantes y negligentes y familias de alto riesgo [Evaluation of a treatment program for abusing and neglecting families and high-risk families]. Intervención Psicosocial, 11(2), 213227.

Bartau Rojas, I. \& de la Caba Collado, M.A. (2009). Una experiencia de mejora de las habilidades para la parentalidad y el desarrollo sociopersonal de los menores en contextos de desprotección social [An experience of improving the skills for parenting and socio-personal development of children in contexts of social vulnerability]. Intervención Psicosocial, 18(2), 135-151.

Barudy, J. \& Dantagnan, M. (2005). Los buenos tratos a la infancia. Parentalidad, apego y resiliencia [Good treatment for children. Parenting, attachment and resilience]. Barcelona: Gedisa.

Barudy, J. \& Dantagnan, M. (2010). Los desafios invisibles de ser madre o padre. Manual de evaluación de competencias y resiliencia parental [The invisible challenges of being a parent. parental resilience and competence assessment manual]. Barcelona: Gedisa.

Barudy, J. \& Marquebreucq, A.P. (2005). Hijas e hijos de madres resilientes [Daughters and children of resilient mothers]. Barcelona: Gedisa.

Baumrind, D. (1971). Current patterns of parental authority. Devolopmental Pyschology, 4, 1-103.

Baumrind, D. (1978). Patrones disciplinarios parentales y la competencia social en los niños [Parental disciplinary patterns and social competence in children]. Juventud y Sociedad, 9(3), 239-276.

Bolivar, A. (2006). Familia y escuela: Dos mundos llamados a trabajar en común [Family and school: Two worlds called to work together]. Revista de Educación, 339, 119-146.

Bornstein, L. \& Bornstein, M.H. (2010). Estilos parentales y el desarrollo social del niño [Parenting styles and child social development]. En R.E. Tremblay, R.G. Barr, R. De Peters \& M.
Boivin (Eds.), Enciclopedia sobre el desarrollo de la primera infancia [en línea] (pp. 1-4). Montreal, Quebec: Centre of Excellence for Early Childhood Development. Recuperado el 1 de enero de 2013 de http://www.enciclope diainfantes.com/documents/Bornstein ESPx p.pdf

Bowlby, J. (1958). A note on mother-child separation as a mental health hazard. British Journal of Medical Psychology, 31(3-4), 247-248.

Bronfenbrenner, U. (1979). The ecology of human development: Experiments in nature and design. Cambridge, MA: Harvard University Press.

Bronfenbrenner, U. (1989). Ecological systems theory. En R. Vasta (Ed.), Annals of child development, Vol. 6 (pp. 187-249). Greenwich, CT: JAI Press.

Bronfenbrenner. U. \& Evans, G.W. (2000). Developmental science in the 21 st century: Emerging theoretical models, research design and empirical findings. Social Development, 9, 115-125.

CEPAL / CELADE (2002). Vulnerabilidad sociodemográfica: Viejos y nuevos riesgos para comunidades, hogares y personas; sintesis y conclusiones, $L C / G$ [Socio-demographic vulnerability: Old and new risks for communities, households and individuals; synthesis and conclusions]. 2170 (SES.29/16). Santiago, Chile.

Cirillo, S. (2012). Malos padres: Modelos de intervención para recuperar la capacidad de ser padre o madre [Bad parents: Intervention models to recover the ability to be a parent]. Buenos Aires: Gedisa.

Cirillo, S. \& Cipolloni, M.V. (1994). L'asistente sociale ruba $i$ bambini [The social worker steals children]. Millán: Raffaello Cortina.

Eraso, J., Bravo, Y. \& Delgado, M. (2006). Creencias, actitudes y prácticas sobre crianza en madres cabeza de familia en Popayán: Un estudio cualitativo [Beliefs, attitudes, and practices about parenting in Popayan mothers: A qualitative study]. Revista de Pediatría, 41(3), 2340.

García-Bacete, F.J. (2003). Las relaciones escuelafamilia: Un reto educativo [School-family relations: An educational challenge]. Infancia $y$ Aprendizaje, 26(4), 425-437. http://dx.doi.org/ $10.1174 / 021037003322553824$ 
Gómez, E., Cifuentes, B. \& Ortún, C. (2012). Padres competentes, hijos protegidos: Evaluación de los resultados del Programa "Viviendo en Familia" [Competent parents, protected children: Evaluation of the results of the "Living in the Family" Program]. Psychosocial Intervention, 21(3), 259-271. http://dx.doi.org/10.5093/ in $2012 \mathrm{a} 23$

Gómez, E. \& Haz, A.M. (2008). Intervención familiar preventiva en programas colaboradores del SENAME: La perspectiva del profesional [Preventive family intervention in SENAME's collaborating programs: The professional perspective]. Psykhé, 17(2), 53-65. http://dx.doi.org/ $10.4067 / \mathrm{s} 0718-22282008000200005$

Gómez, E. \& Kotliarenco, M.A. (2010). Resiliencia familiar: Un enfoque de investigación e intervención con familias multiproblemáticas [Family resilience: A research and intervention approach with multiproblem families]. Revista de Psicología, 19(2), 43-54.

Gómez, E. \& Muñoz, M. (2012). Efectos de la terapia de interacción guiada sobre el bienestar de díadas en riesgo psicosocial [The effects of guided interaction therapy on the well-being of dyads at psychosocial risk]. Terapia Psicológica, 30(1), 15-24.

Gómez, E., Muñoz, M.M. \& Haz, A.M. (2007). Familias multiproblemáticas y en riesgo social: Características e intervención [Multiproblem families and social risk: Characteristics and intervention]. Psykhe, 16(2), 43-54. http://dx. doi.org/10.4067/S0718-22282007000200004

Gómez, E., Muñoz, M.M. \& Santelices, M.P. (2008). Efectividad de las intervenciones en apego con infancia vulnerada y en riesgo social: Un desafío prioritario para Chile [Effectiveness of attachment interventions with children at risk and at social risk: A priority challenge for Chile]. Terapia Psicológica, 26(2), 241-251.

Gracia, E. (1997). El apoyo social en la intervención comunitaria [The social support in community intervention]. Barcelona: Paidós.

Gracia, E. \& Herrero, J. (2006). La comunidad como fuente de apoyo social: Evaluación e implicaciones en los ámbitos individual y comunitario [The community as a source of social support: Evaluation and implications at the indiv- idual and community levels]. Revista Latinoamericana de Psicología, 38, 327-342.

Gracia, E. \& Musitu, G. (2000). Psicología social de la familia [Social psychology of the family]. Barcelona: Paidós.

Grusec, J.E. (2002). Parental socialization and children's acquisition of values. En M.H. Bornstein (Ed.), Handbook of parenting, Vol. 5 (pp. 143-167). Mahwah: Erlbaum.

Grusec, J.E. \& Goodnow, J. (1994). Impact of parental discipline methods on the child's internalization of values: A reconceptualization of current points of view. Developmental Psychology, 30(1), 4-19.

Hidalgo García, M.V., Menéndez Álvarez-Dardet, S., Sánchez Hidalgo, J., Lorence Lara, B. \& Jiménez García, L. (2009). La intervención con familias en situación de riesgo psicosocial: Aportaciones desde un enfoque psicoeducativo [Intervention with families at a psychosocial risk: Contributions from a psychoeducational approach]. Apuntes de Psicología, 27(2-3), 413426.

Izzedin Bouquet, R. \& Pachajoa Londoño, A. (2009). Pautas, prácticas y creencias acerca de crianza. Ayer y hoy [Guidelines, practices, and beliefs about parenting. Yesterday and today]. Liberabit, 15(2), 109-115.

Jiménez, L. (2009). Crecer en familias en situación de riesgo. Análisis evolutivo durante la infancia $y$ adolescencia [Grow in families at risk. Evolutionary analysis during childhood and adolescence]. Tesis doctoral no publicada. Universidad de Sevilla, España.

Kotliarenco, M., Muñoz, M., Gómez, E. \& Armijo, I. (2009). Evaluación pre-post del desarrollo psicomotor y el estilo de apego en los usuarios de los centros de desarrollo infantil temprano [Pre-post evaluation of psychomotor development and attachment style in users of early childhood development centers]. Summa Psicológica, 6(2), 89-104.

Krumm, G., Vargas Rubilar, J. \& Gullón, S. (2013). Estilos parentales y creatividad en niños escolarizados [Parenting styles and creativity in school children]. Psicoperspectivas, 12(1), 153-173.

Lecannelier, F., Flores, F., Hoffmann, M. \& Vega, T. (2010). Trayectorias tempranas de la agre- 
sión: Evidencias y la propuesta de un modelo preventivo. En D. Sirpolu \& H. Salgado (Eds.), Trayectorias [Trajectories]. Santiago. Chile: Universidad del Desarrollo.

Maccoby, E.E. (1992). The role of parents in the socialization of children. An historical review. Developmental Psychology, 28, 1006-1020.

Máiquez Chaves, M.L. \& Capote Cabrera, C. (2001). Modelos y enfoques en intervención familiar [Models and approaches in family intervention]. Intervención Psicosocial, 10(2), 185198.

Martín Quintana, J.C., Máiquez Chaves, M.L., Rodrigo López, M.J., Byrne, S., Rodríguez Ruiz, B. \& Rodríguez Suárez, G. (2009). Programas de educación parental [Parental education programs]. Intervención Psicosocial, 18, 121-133.

Méndez-Castellano, H. \& Méndez, M.C. (1994). Sociedad y estratificación, método GraffarMéndez Castellano [Society and stratification, method Graffar-Méndez Castellano]. Caracas: Fundacredesa.

Muñoz Silva, A. (2005). La familia como contexto de desarrollo infantil. Dimensiones de análisis relevantes para la intervención educativa y social [The family as a child development context. Dimensions of analysis relevant to educational and social intervention]. Portularia, 5 (2), 147-163.

Nuño-Gutiérrez, B.L., Alvarez-Nemegyei, J., Madrigal de León, E., Martínez Becerra, B.A. \& Miranda Moreno, R. (2006). Efectos a corto plazo de un programa educativo "Escuela para padres" sobre el ambiente familiar. [Short-term effects of an educational program "School for parents" on the family environment]. Revista Médica del Instituto Mexicano del Seguro Social, 44(6), 519-527.

Palacios, J. (1999). La familia como contexto de desarrollo humano [The family as a context of human development]. Sevilla: Secretariado de Publicaciones de la Universidad de Sevilla.

Palacios, J. \& Rodrigo, M. (1998). La familia como contexto de desarrollo humano. En M.J. Rodrigo \& J. Palacios (Coords.), Familia y desarrollo humano [Family and human development] (pp. 24-44). Madrid: Alianza Editorial.
Peña, M., Máiquez, M.L. \& Rodrigo, M.J. (2014). Efectos de la inclusión de contenidos de desarrollo personal en un programa de educación parental para familias en riesgo psicosocial [Effects of inclusion of personal development content in a parental education program for families at psychosocial risk.]. Anales de Psicología, 30(1), 201-210. https://doi.org/10.601 8/analesps.30.1.152331

Pereira, P., D'Affonseca, S.M. \& Williams, L.C.A. (2013). A feasibility pilot intervention program to teach parenting skills to mothers of polyvictimized children. Journal of Family Violence, 28, 5-15. https://doi.org/10.1007/s10896012-9490-9

Pérez Campos, G. (2005). La complejidad de los marcos de interacción en educación de padres. [The complexity of parents' interaction frameworks in education]. Avances en Psicología Latinoamericana, 23, 177-192.

Pérez Cárdenas, C.P. \& Fernández Benítez, O.C. (2005). Evaluación de un programa de intervención familiar educativa [Evaluation of an educational family intervention program]. $R e$ vista Cubana de Medicina General Integral, 21(1-2) [on line].

Pérez Padilla, J., Menéndez Álvarez-Dardet, S. \& Hidalgo, M.V. (2014). Estrés parental, estrategias de afrontamiento y evaluación del riesgo en madres de familias en riesgo usuarias de los Servicios Sociales [Parental stress, coping strategies, and risk assessment in mothers of atrisk families would use social services]. Psychosocial Intervention, 23(1), 25-32. https:// doi.org/10.5093/in2014a3

Rey, C. (2006). Entrenamiento de padres: Una revisión de sus principales componentes y aplicaciones [Parent training: A review of its major components and applications]. Revista Infancia, Adolescencia y Familia, 1, 61-84.

Richaud de Minzi, M.C. (2006). Loneliness and depression in middle and late childhood: Its relationship to attachment and parental styles. Journal of Genetic Psychology, 167(2), 189210.

Richaud de Minzi, M.C. (2007). Parental styles and attachment in relation with self control, social skills and coping in children at risk for poverty. 
En D.M. Devor (Ed.), New developments in parent-child relations (pp. 87-110). Hauppauge, NY: Nova Editorial Publishers.

Richaud, M.C. (2013). Contributions to the study and promotion of resilience in socially vulnerable children. American Psychologist, 68(1), 751-758. http://dx.doi.org/10.1037/a0034327

Richaud, M.C. \& Arán Filippetti, V. (En prensa). Children's cognitive development in social vulnerability: An interventional experience. Jour nal of Psychology Research.

Richaud, M.C., Lemos, V. \& Vargas Rubilar, J. (2014). Argentine culture and parenting styles. En H. Selin (Ed.), Parenting across culture: Motherhood and fatherhood in non-western cultures. Series: Science across cultures: The history of non-western science, Vol. 7 (pp. 277-292). Springer Publishers of The Netherlands.

Rivas Borrel, S. \& Ugarte Artal, C. (2014). Formación docente y cultura participativa del centro educativo: Claves para favorecer la participación familia-escuela [Teacher training and participatory culture of the school: Keys to fostering family-school participation]. Estudios sobre Educación, 24, 153-168.

Rodrigo, M.J. \& Byrne, S. (2011). Apoyo social y agencia personal en madres en situación de riesgo [Social support and personal agency in mothers at risk]. Intervención Psicosocial, 20 (1), 13-24.

Rodrigo, M.J., Máiquez, M.L., Martín, J.C. \& Byrne, S. (2008). Preservación familiar: Un enfoque positivo para la intervención con familias [Family preservation: A positive approach to intervention with families]. Madrid: Pirámide.

Rodrigo López, M.J., Martín Quintana, J.C., Cabrera Casimiro, E. \& Máiquez Chaves, M.L. (2009). Las competencias parentales en contextos de riesgo psicosocial [Parental competences in psychosocial risk contexts]. Intervención Psicosocial, 18(2), 113-120.

Sanders, M.R. \& Morawska, A. (2010). ¿Es posible que el conocimiento de los padres, las competencias y expectativas disfuncionales y la regulación emocional mejoren los resultados de los niños? [Is it possible for parents' know- ledge, dysfunctional skills and expectations, and emotional regulation to improve children's outcomes?]. En R.E. Tremblay, R.G. Barr, R. De V. Peters \& M. Boivine (Eds.), Enciclopedia sobre el desarrollo de la primera infancia [en línea] (pp. 1-13). Montreal, Quebec: Centre of Excellence for Early Childhood Development. Recuperado el 3 de febrero de 2013 de http://www.enciclopedia-infantes.com/docu ments/Sanders-MorawskaES Px p.pdf

Sanders, M.R. \& Ralph, A. (2004).Towards a multi-level model of parenting intervention. En M. Hoghughi \& N. Long (Eds.), Handbook of parenting: Theory and research for practice (pp. 352-368). London, England: Sage.

Sousa, L., Ribeiro, C. \& Rodrigues, S. (2006). Intervention with multi-problem poor clients: Towards a strengths-focused perspective. Journal of Social Work Practice, 20(2), 189-204. https://doi.org/10.1080/02650530600776913

Torío López, S., Peña Calvo, J.V., Rodríguez Menéndez, M.C., Fernández García, C.M. \& Molina Martín, S. (2010). Hacia la corresponsabilidad familiar: Construir en lo cotidiano. Un programa de educación parental [Towards family co-responsibility: Building in the every day. A parental education program]. Educatio Siglo XXI, 28(1), 85-108.

Trivette, C. \& Dunst, C. (2010). Programas comunitarios de apoyo parental [Community support programs for parental support]. Enciclopedia sobre el desarrollo de la primera infancia [en línea] (pp. 1-8). Orelena Hawks Puckett Institute, EE.UU. Recuperado el 10 de febrero de 2013 de http://www.enciclopedia-infantes.co $\mathrm{m}$ /documents/Trivette-DunstESPx p.pdf

Vargas Rubilar, J. (2009). Percepción de clima social familiar y actitudes ante situaciones de agravio en la adolecencia tardía [Perception of family social climate and attitudes to grievance situations in late adolescence]. Interdisciplinaria, 26(2), 289-316.

Vargas Rubilar, J. (2014). Un estudio de parentalidad en contextos de vulnerabilidad social [A study of parenting in contexts of social vulnerability]. Tesis Doctoral no publicada. Pontificia Universidad Católica Argentina. Buenos Aires, Argentina. 
Vargas Rubilar, J. \& Arán Filippetti, V. (2014). Importancia de la parentalidad en el desarrollo cognitivo infantil: Una revisión teórica [The importance of parenting for the child's cognitive development: A theoretical revision]. Revista Latinoamericana de Ciencias Sociales, Niñez y Juventud, 12(1), 176-181.

Vargas Rubilar, J. \& Oros, L. (2011). Una propuesta de intervención psicoeducativa para promover la autoestima infantil [A proposal for psychoeducational intervention to promote child self-esteem]. Revista Acta Psiquiátrica y Psicológica de América Latina, 57(3), 235-244.
Vargas Rubilar, J., Oros, L. \& Richaud, M.C. (Inédito). Program to promote positive parenting: Results from the perspective of participating mothers.

Walsh, F. (2004). Resiliencia familiar: Estrategias para su fortalecimiento [Family resilience: Strategies for strengthening]. Buenos Aires: Amorrortu.

Weissbourd, B.A. \& Kagan, S.L. (1989). Family support programs: Catalysts for change. American Journal of Orthopsychiatry, 59, 20-31.

Winnicott, D.W. (2006). La familia y el desarrollo del individuo [The family and the development of the individual]. Buenos Aires: Ediciones Hormé.

Grupo Vinculado al Centro Interdisciplinario de Investigaciones en Psicología Matemática y Experimental (CIIPME)

Universidad Adventista del Plata (UAP)

Consejo Nacional de Investigaciones Cientificas y Técnicas (CONICET)

Diamante, Provincia de Entre Rios República Argentina

Fecha de recepción: 10 de marzo de 2016 Fecha de aceptación: 29 de diciembre de 2016 